\title{
Analysis of the chronic intake of and withdrawal from diazepam on emotional reactivity and sensory information processing in rats
}

De Ross, J; Castilho, V. M., Brandão, M. L; Nobre M. J.

Instituto de Neurociências \& Comportamento - INeC

Campus USP, Ribeirão Preto, SP, Brasil, 14040-901.

Laboratório de Psicobiologia, Departamento de Psicologia,

FFCLRP, Campus USP, Ribeirão Preto, São Paulo, Brasil, 14040-901. 


\section{Abstract}

It has been demonstrated that, on abrupt withdrawal, patients with chronic exposure can experience a number of symptoms indicative of a dependent state. In clinical patients, the earliest to arise and most persistent signal of withdrawal from chronic benzodiazepine (Bzp) treatment is anxiety. In laboratory animals, anxiety-like effects following abrupt interruption of chronic Bzp treatment can also be reproduced. In fact, signs that oscillate from irritability to extreme fear behaviours and seizures have been described already. As anxiety remains one of the most important symptoms of Bzp withdrawal, in this study we evaluated the anxiety levels of rats withdrawn from diazepam. Also studied were the effects on the motor performance and preattentive sensory gating process of rats under diazepam chronic treatment and upon 48-h withdrawal on three animal models of anxiety, the elevated plus-maze (EPM), ultrasonic vocalizations (USV) and startle + prepulse inhibition tests. Data obtained showed an anxiolytic- and anxiogenic-like profile of the chronic intake of and withdrawal from diazepam regimen in the EPM test, 22-KHz USV and startle reflex. Diazepam chronic effects or its withdrawal were ineffective in promoting any alteration in the prepulse inhibition (PPI). However, an increase of PPI was achieved in both sucrose and diazepam pretreated rats on 48-h withdrawal, suggesting a procedural rather than a specific effect of withdrawal on sensory gating processes. It is also possible that the prepulse can function as a conditioned stimulus to informing the delivery of an aversive event, as the auditory startling-eliciting stimulus. All these findings are indicative of a sensitization of the neural substrates of aversion in diazepam withdrawn animals without concomitant changes on the processing of sensory information.

Keywords: Anxiety, Diazepam withdrawal, Elevated-plus-maze, Prepulse inhibition, Ultrasonic vocalization.

Abbreviations: Bzp, benzodiazepines; EPM, elevated plus-maze test; PPI, prepulse inhibition;

USV, ultrasonic vocalizations. 


\section{Introduction}

It is well recognized that, in addition to its peculiar somatic signs, withdrawal from most drugs of abuse including alcohol, psychostimulants and nicotine, opiates and benzodiazepines (Bzp) can promote common disturbances in affective states and emotionality (Markou et al., 1998). In the case of Bzp, chronic treatment was supposed not to cause dependence. However, it has been demonstrated that, on abrupt withdrawal, patients with chronic exposure can experience a number of symptoms indicative of a dependent state. The earliest to arise and most persistent signal of withdrawal from chronic Bzp treatment is anxiety (Marks, 1978), although clinical reports have also denounced the appearance of high levels of fear in Bzp withdrawn patients (Rosebush and Mazurek, 1996).

In laboratory animals, similar aspects of the withdrawal phenomena can be reproduced. In fact, signs that oscillate from irritability to extreme fear behaviours and seizures have already been described (Ashton, 2005; Emmett-Oglesby et al., 1983a; Lacerra et al., 1999; Owen and Tyrer, 1983; Petursson and Lader, 1981a, 1981b; Woods et al., 1987). For example, rats withdrawn from diazepam exhibited high levels of aversion when submitted to the elevated plus-maze (EPM) (File and Andrews, 1991; Martijena et al., 1996), increased levels of ultrasonic vocalizations (USV) on 22-KHz frequencies and enhanced startle response amplitude to an auditory startling-eliciting stimulus (Vivian et al., 1994; Vivian and Miczek, 1993). A similar level of defensive behaviour is observed in animals faced with several dangerous situations such as exposure to predators (Blanchard et al., 1991), foot-shocks (De Vry et al., 1993) or playback of 22-KHz aversive frequencies (Brudzynski and Chiu, 1995). However, in most of the studies cited above chronic intraperitoneal injection, a procedure known to 
enhance anxiety levels of rats (Griebel et al., 1994), was used as the choice of drug delivery.

The amplitude of startle reflex response is attenuated when the startling-eliciting stimulus, as an auditory one, is preceded by a weak prepulse non-startling stimulus. This phenomenon is termed prepulse inhibition (PPI) and has been observed across a wide range of species (Geyer and Ellenbroek, 2003; Hoffman and Ison, 1980). However, the influence of the anxiolytic effects of chronic treatment with diazepam or the aversive effects of withdrawal from this Bzp on the processing of a prepulse signal has not yet been investigated.

Based on these assumptions, the present study was designed to provide a broad perspective on the potential influence of chronic effects of and withdrawal from oral intake of diazepam on emotional reactivity as well as on the processing of sensory information in rats. The first two parts of this study were designed to assess the effectiveness of chronic oral treatment with diazepam and its withdrawal in promoting anxiolytic and aversive effects, respectively, in animals tested on the EPM test and 22KHz USV elicited by foot-shock. In the third part, acoustic startle responses and PPI were assessed with the same approach, as these measures have been used to index motor performance and sensory-motor gating, respectively.

\section{Materials and methods}

\subsection{Animals}

One hundred and sixteen male Wistar rats, weighing 100-110 $\mathrm{g}$ at the beginning of the treatment, from the animal house of the campus of Ribeirão Preto, University of São Paulo, were used. They were housed in groups of four in Plexiglas-walled cages, lined with wood shavings that were changed every three days, maintained in a 12:12 
dark/light cycle (lights on 07:00 h) at $24 \pm 1^{\circ} \mathrm{C}$. The rats were given free access to food and partial access to water. Before the beginning of the treatments, the animals had a three-day habituation period to the lodging conditions, deprivation and drinking procedures. The experiments reported in this article were performed in compliance with the recommendations of $\mathrm{SBNeC}$ (Brazilian Society for Neuroscience and Behavior), which are in accordance with the rules of the U.S. National Institutes of Health Guide for Care and Use of Laboratory Animals.

\subsection{Administration of diazepam by oral route}

The oral intake procedure as the choice for drug administration was chosen mainly to avoid the anxiogenic-like effects promoted by chronic systemic drug injections (Griebel et al., 1994). Moreover, in a previous study by our laboratory (data not published yet), we showed that rats receiving 18 days of chronic i.p. saline injections displayed high anxiety levels when compared with animals that received chronic oral sucrose or those that remained undisturbed in the vivarium and were tested on plus-maze. This demonstrated that the oral drug administration procedure is supposed to produce more precise data than those approaches using i.p. injections.

The procedure used for diazepam oral intake has been described previously (Fontanesi et al., 2007). Briefly, the animals were submitted on a daily basis to 14 hours of water deprivation (19:00 to 9:00), followed by 10 hours of water ad libitum (9:00 to 19:00). This procedure began three days before the start of the 18-day treatment, as an acclimitization period for the animals to the drinking procedure. Diazepam was separately dissolved in a concentration of $10 \mathrm{mg} / \mathrm{ml}$ of saline plus propilenoglycol (5\%) and offered, once daily, in a dose of $1 \mathrm{ml} / \mathrm{kg}$ diluted in a solution of $2 \mathrm{ml}$ of tap water added to sucrose (5\%) plus propilenoglycol (5\%), which was also used as control 
solution. The solutions were made available to the animals in glass pipettes of $5 \mathrm{ml}$, at the end of the daily period of water deprivation $(09: 00 \mathrm{~h})$, and were delivered during each of the 18 days of treatment. Except for the first two days of treatment, in which control and experimental solutions were available for 30 minutes, the animals that did not drink the sucrose or diazepam solutions for 10 minutes were removed from the experiments. This was necessary to avoid prolonging the deprivation period. Experimental sessions were conducted for 30 minutes (termed the chronic effects condition, in which the animals were tested during the effect of the drug) or 48 hours after the last oral drug intake (the withdrawal condition, in which the animals were tested free of the drug).

\subsection{Experiment I}

\section{Withdrawal-induced anxiety in rats tested in the EPM}

\subsubsection{Experimental apparatus}

This experiment was designed to observe the influence of chronic intake of diazepam or its interruption on the anxiety levels of rats tested in the EPM. The EPM was made of wood and had two open arms $(50 \times 10 \mathrm{~cm})$, perpendicular to two closed arms of equal dimensions, surrounded by $40-\mathrm{cm}$ walls. The apparatus was elevated 50 cm from the floor (Anseloni and Brandão, 1997). To avoid falls, a 3-mm wooden rim surrounded the open arms. The EPM was located inside a refrigerated room with a constant noise $(50 \mathrm{~dB})$. Behaviour in the EPM was documented by a camera (Everfocus, Duarte, CA, USA) linked to a monitor and video-cassette, outside the experimental room, which allowed the recordings to be analysed later. Luminosity at the level of the open arms of the maze was 20 lux. Four groups of animals were arranged in order to avoid the same animal being exposed twice to the maze ( $n=12$ for each group). The 
groups were named sucrose chronic effects, diazepam chronic effects, sucrose withdrawal and diazepam withdrawal.

\subsubsection{Experimental procedure}

The rat was placed at the centre of the EPM facing an enclosed arm and allowed to explore the environment for 5 minutes. The apparatus was cleaned with $20 \%$ ethanol and water before the next rat was placed in the EPM. The measures taken were the traditional indexes of exploration expressed as percentage of entries (with the four paws) into and time spent on the open arms in relation to total number of entries and time, respectively. The total number of entries into the closed arms was also recorded. Animals of the sucrose or diazepam groups were tested for 30 minutes or 48 hours after the last sucrose or diazepam oral intake, respectively. Each animal was tested only once.

\subsubsection{Statistical analysis}

The data obtained in the EPM were analysed using one-way ANOVA with the treatments (sucrose or diazepam) as the independent factor, and the number of closed arm entries, percentage of entries and time spent in the open arms as the dependent factor. Newman-Keuls post-hoc comparisons were carried out whenever significant overall F-values were obtained. In all cases a probability level of $p<0.05$ was considered to be significant.

\subsection{Experiment II}

Effects of diazepam withdrawal on USV induced by foot-shocks

\subsubsection{Experimental apparatus}


The apparatus used for recording and analysing the USV has been described in detail in previous reports (Bassi et al., 2007; Nobre et al., 2003; Nunes Mamede Rosa et al., 2005). Briefly, it consisted of a testing box $(25 \times 15 \times 12 \mathrm{~cm})$ made of steel bars spaced approximately $12 \mathrm{~mm}$ apart. This experimental chamber was situated inside a larger, padded, echo-free (sound-attenuated) and ventilated chest $(60 \times 40 \times 45 \mathrm{~cm})$ with a $28 \mathrm{~W}$ red light bulb located at the top of the chamber. For recording and analysis of USV, four ultrasound bat detectors (Ultra Sound Advice, London, UK) were mounted in the lateral and rear walls of the chamber and linked to a multichannel audio apparatus with 20-KHz low band pass (cut-off) filter (Noldus Instruments, Amsterdam, The Netherlands) and then fed into a computer. During each testing session each bat detector was tuned to a different frequency $(20,22,24$ or $26 \mathrm{KHz})$ to record the USV.

The choice of the frequencies above was based on previous studies which showed that rats exposed to dangerous situations display an enhancement of the emissions of 22- and 24-kHz USV that do not differentiate from each other (Nobre and Brandão, 2003; Nunes Mamede Rosa et al., 2005). The same studies showed that the 20- and $26-\mathrm{kHz}$ frequencies are emitted less. The sessions consisted of placing the animals individually inside the experimental chamber for 5 minutes of acclimitization. After this, they received just one foot-shock $(0.6 \mathrm{~mA}, 1 \mathrm{~s})$, produced by a shock generator (Insight, Sao Paulo, Brazil). A video-camera linked to a TV monitored the behaviour for the overall 10 minutes period of recording after the foot-shock was delivered. The number of calls and duration of USV emission in seconds were analysed by a sonograph (Ultravox signal analysis work station, Noldus Instruments, Amsterdam, The Netherlands), stored on a hard disc and subsequently transferred to tables in a spreadsheet programme (Excel; Microsoft Corp., Mountain View, CA, USA) for off- 
line analysis. Each frequency input in each of the four microphones used was calibrated before the session began with the use of an audio generator (GW, $1 \mathrm{MHz}, \mathrm{NJ}, \mathrm{USA})$.

\subsubsection{Experimental procedure}

Two independent groups of animals were used to evaluate the effects of diazepam withdrawal on foot-shock-induced ultrasonic vocalizations. As in experiment I, the animals were submitted to an 18-day chronic intake of sucrose or diazepam solutions ( $\mathrm{n}=14$ in each group). Twenty minutes after the last sucrose or diazepam intake the animals were handled for 5 minutes and then placed in the experimental box, where they remained undisturbed for an additional 5 minute period of acclimitization. Immediately after the foot-shock was delivered ultrasonic measures were registered for 10 minutes (chronic sessions). Forty-eight hours later, using the same protocols, the same animals were submitted to the withdrawal sessions.

\subsubsection{Statistical analysis}

Data are reported as mean + SEM. Separate two-way repeated measures (RM) ANOVA for comparisons between values recorded 30 minutes or 48 hours after withdrawal were performed, with the treatments as the independent variables and the frequency as the dependent repeated variable. In addition, comparisons were carried out to evaluate the differences between the exposure to chronic and withdrawal effects in the same group (sucrose or diazepam). Post-hoc comparisons were carried out whenever significant overall F-values were obtained. A value of $\mathrm{p}<0.05$ was considered significant. 


\subsection{Experiment III}

\section{Effects of diazepam chronic intake and withdrawal on startle response and PPI}

\subsubsection{Experimental apparatus}

The rats were placed in a stabilimeter, which consisted of a wire-mesh cage $(16.5 \times 5.1 \times 7.6 \mathrm{~cm})$ suspended within a PVC frame $(25 \times 9 \times 9 \mathrm{~cm})$, which was attached to the response platform with four thumbnail-screws. The stabilimeter and platform were located inside a ventilated plywood sound-attenuating chamber $(64 \times 60$ $\times 40 \mathrm{~cm}$ ). The floor of the stabilimeter consisted of six stainless steel bars $3.0 \mathrm{~mm}$ in diameter and spaced $1.5 \mathrm{~cm}$ apart.

The startle reaction of the rats generated a pressure on the response platform and analogue signals were amplified, digitized and analysed by the software of the startle measurement system (Insight, Sao Paulo, Brazil), which also controlled all parameters of the session (intensity of the acoustic stimulus, inter-stimulus interval, etc.). The startle amplitude was recorded within a time window of $200 \mathrm{~ms}$ after the onset of the startle stimulus. Two loudspeakers located $10 \mathrm{~cm}$ above the floor, on each lateral side of the acoustic isolation chamber, were used to deliver the prepulse stimulus, the acoustic startle stimuli and a continuous background noise (white noise, $50 \mathrm{~dB}$ ).

Calibration procedures were conducted before the experiments to ensure equivalent sensitivities of the response platforms over the test period. Animal behaviour was recorded by a video camera (Safety View, São Paulo, Brazil) located behind the stabilimeter, allowing the discrimination of all possible behaviours, with the signal being relayed to a monitor in another room via a closed circuit. A red light bulb (15 W) was located on top of the experimental box, $50 \mathrm{~cm}$ above the stabilimeter, to provide illumination for the camera. 


\subsubsection{Experimental procedure}

Startle response and PPI was tested in a simple paradigm routinely used in our laboratory (Cabral et al., 2006; Nunes Mamede Rosa et al., 2005). The acoustic startle test session for measuring the PPI consisted of four parts. The first was a 5 minutes period of acclimitization to the startle test chamber. No acoustic startle stimuli were presented during this period. The second part consisted of 20 presentations of a pulse alone (70 $\mathrm{dB}$ of pure tone, $3,000 \mathrm{~Hz}$ frequency, $20 \mathrm{~ms}$ duration). The third part consisted of 20 presentations of an acoustic startle stimulus (pulse, 110-dB, 50-ms bursts of white noise having a rise-decay time of $5 \mathrm{~ms}$ ). The fourth part tested the prepulse modulation of the acoustic startle (prepulse + pulse). It consisted of 20 trials of an acoustic startle stimuli preceded $30 \mathrm{~ms}$ by a pure tone (prepulse) with a 30 seconds inter-trials interval.

In this study, a pure tone was used as a prepulse stimulus since we have noted that the use of a white noise, but not a pure tone, activates both the affective and sensory channels, as this type of sound seems to promote aversion. The purpose of the procedures above was to determine the effects of the acclimitization to the continuous presentation of the prepulse, startle or prepulse + startle stimuli on the amplitude of startle responses. The inter-stimulus interval of $30 \mathrm{~s}$ used between trials was based on a previous study by our laboratory (Cabral et al., 2006; Nunes Mamede Rosa et al., 2005).

The testing session lasted 35 minutes. The startle response amplitudes obtained from this experiment were stored on a hard disc and subsequently transferred to tables in a spreadsheet programme for off-line analysis. Two groups were formed: sucrose (n $=21)$ and diazepam $(n=19)$. The same animals were tested 30 minutes (during chronic drug effects) and $48 \mathrm{~h}$ after the last diazepam oral intake (during withdrawal effects). 


\subsubsection{Statistical analysis}

The results from startle and PPI tests are all reported as means \pm SEM. Startle responses were averaged for each animal across the entire session and used as the data for statistical analysis. A two-way RM ANOVA was conducted on the startle and PPI, separately, with the groups (sucrose $\mathrm{x}$ diazepam) as the independent and the amplitude of startle responses obtained during chronic or withdrawal conditions as the dependent repeated factors. The results of the PPI are reported as \% PPI $=100-(100 \times$ mean startle magnitude on 'pre-pulse + pulse' stimulus divided by the mean startle magnitude on 'pulse' alone). All statistical analyses were followed, when appropriate, by NewmanKeuls test. $\mathrm{P}<0.05$ was considered to be significant.

\section{Results}

\subsection{Withdrawal-induced anxiety in rats tested in the EPM}

One-way ANOVA showed significant differences on the percentage of entries $\left(F_{3,44}=13.66, p<0.0001\right)$ and time spent in the open arms $\left(F_{3,44}=16.52, p<0.0001\right)$, and on the number of closed arms entries $\left(\mathrm{F}_{3,44}=3.65, \mathrm{p}<0.05\right)$. Post-hoc analysis revealed that rats tested 30 minutes after the last chronic oral intake of diazepam showed little anxiety-like behaviour (Figure 1) when compared with the control group, as revealed by the increase in the mean percentage of time spent in the open arms displayed by these animals $\left(\mathrm{F}_{3,44}=9.86, \mathrm{p}<0.0001\right)$. On the other hand, $48 \mathrm{~h}$ of diazepam withdrawal enhanced the levels of anxiety-like behaviour as revealed by the reduction in both the percentage of entries and time spent in the open arms of the EPM. One-way ANOVA analysis also demonstrated a significant effect of the treatments on the number of closed arms entries $\left(F_{3,44}=3.65, p<0.05\right)$. Post-hoc test revealed that this 
effect was due to the ability of diazepam to increase the motor behaviour of rats tested under the effects of this drug (chronic sessions).
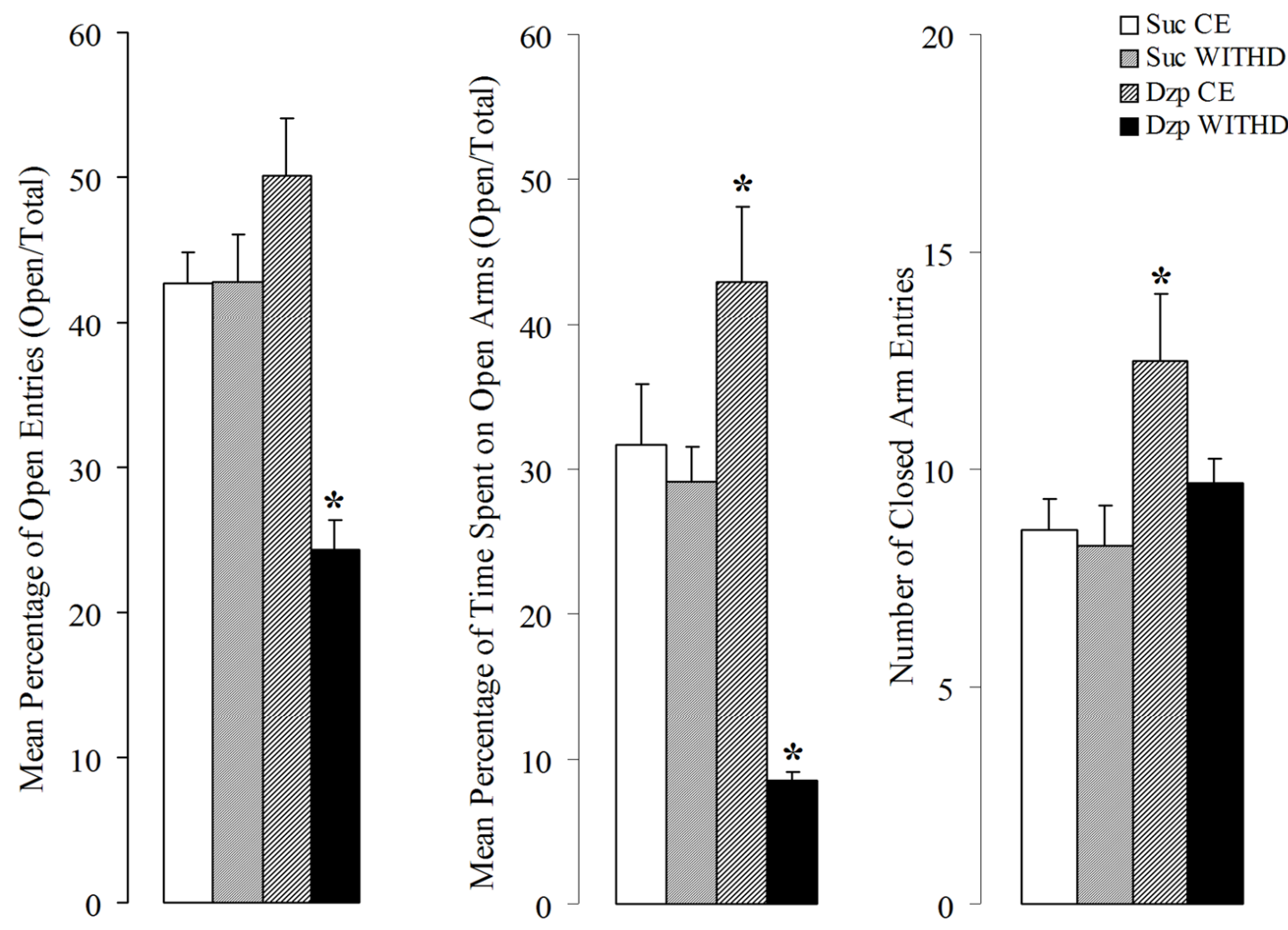

Fig 1

Figure 1: Mean (+SEM) percentage of entries and time spent in the open arms, and mean number of closed arms entries of rats chronic treated with (Suc) or diazepam (Dzp) and tested 30 min (chronic effects - CHRON) after the last oral intake or on $48 \mathrm{~h}$ withdrawal (WITHD). * Significant difference between Suc or Dzp groups when compared with their respectively CHRON or WITHD conditions. Oneway ANOVA followed by Newman-Keuls post-hoc.

\subsection{Effects of diazepam withdrawal on USV induced by foot-shocks}

Figures 2 and 3 show the mean number and duration of USV emitted at the frequencies of 20,22, 24 and $26 \mathrm{KHz}$ soon after foot-shocks were delivered 30 minutes (chronic condition) or $48 \mathrm{~h}$ (withdrawal condition) after the interruption of the 
treatments with sucrose or diazepam oral intake. The number and duration of USV at 20 to $26 \mathrm{KHz}$ was subjected, independently, to a two-way RM ANOVA (treatment $\times$ frequency or condition $\times$ frequency) with the frequencies as the repeated measures.

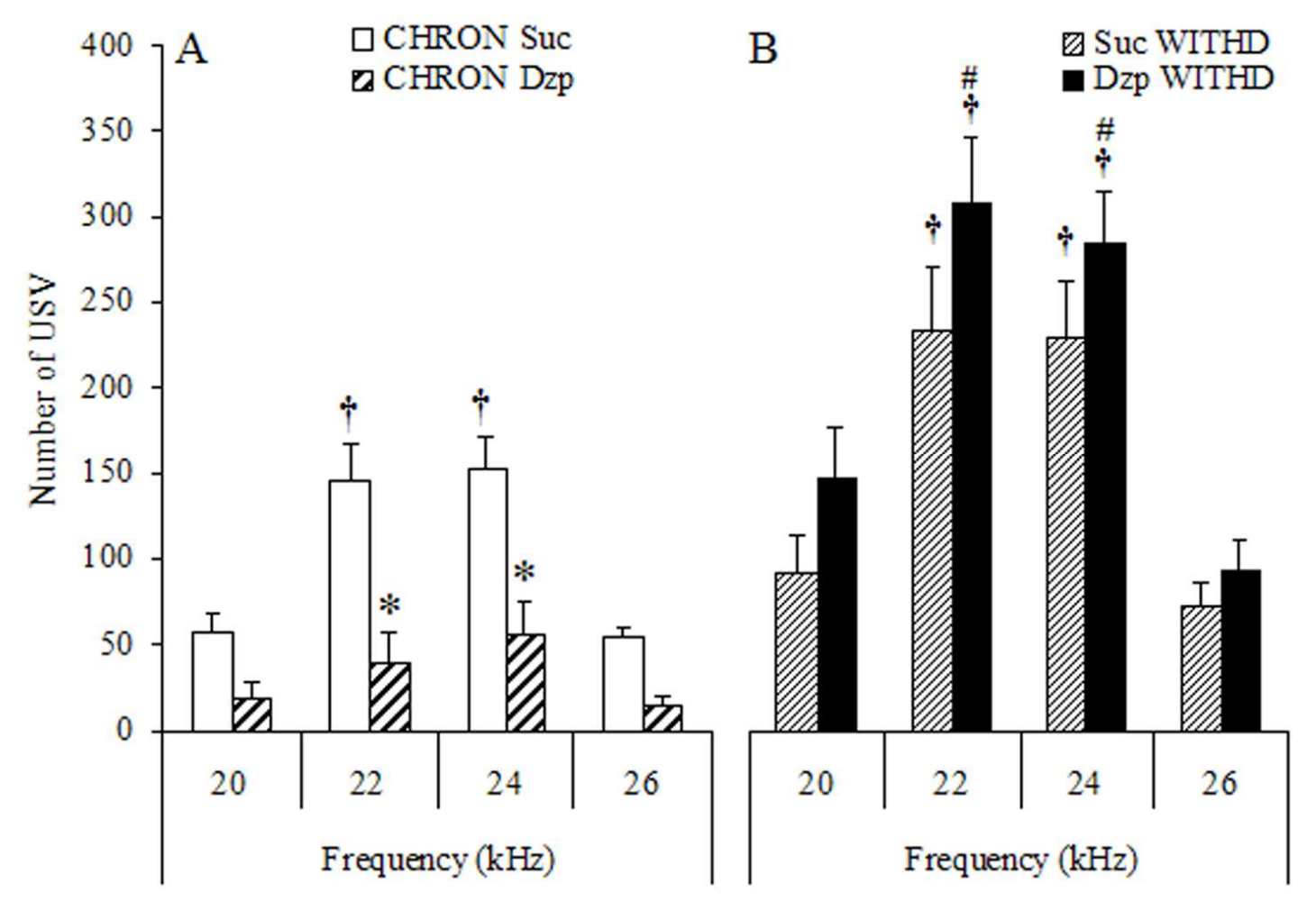

Figure 2: Mean (+SEM) of the number of USV emitted at the frequencies of $20-26 \mathrm{KHz}$ during the first $10 \mathrm{~min}$ after foot-shock delivery in rats tested $30 \mathrm{~min}$ after the last drug intake (2A, CHRON) or $48 \mathrm{~h}$ withdrawal (2B, WITHD) from sucrose (Suc) or diazepam (Dzp). *Significant difference between Dzp and Suc pretreated animals in the same frequency on the same condition CHRON or WITHD). $†$ Significant difference between frequencies on the same group (Suc or Dzp) and condition (CHRON or WITHD) when compared with the $20 \mathrm{KHz}$ frequency. \#Significant difference between WITHD or CE conditions on the same frequency on the same group (Dzp or Suc). Two-way RM ANOVA followed by the Newman-Keuls test. 


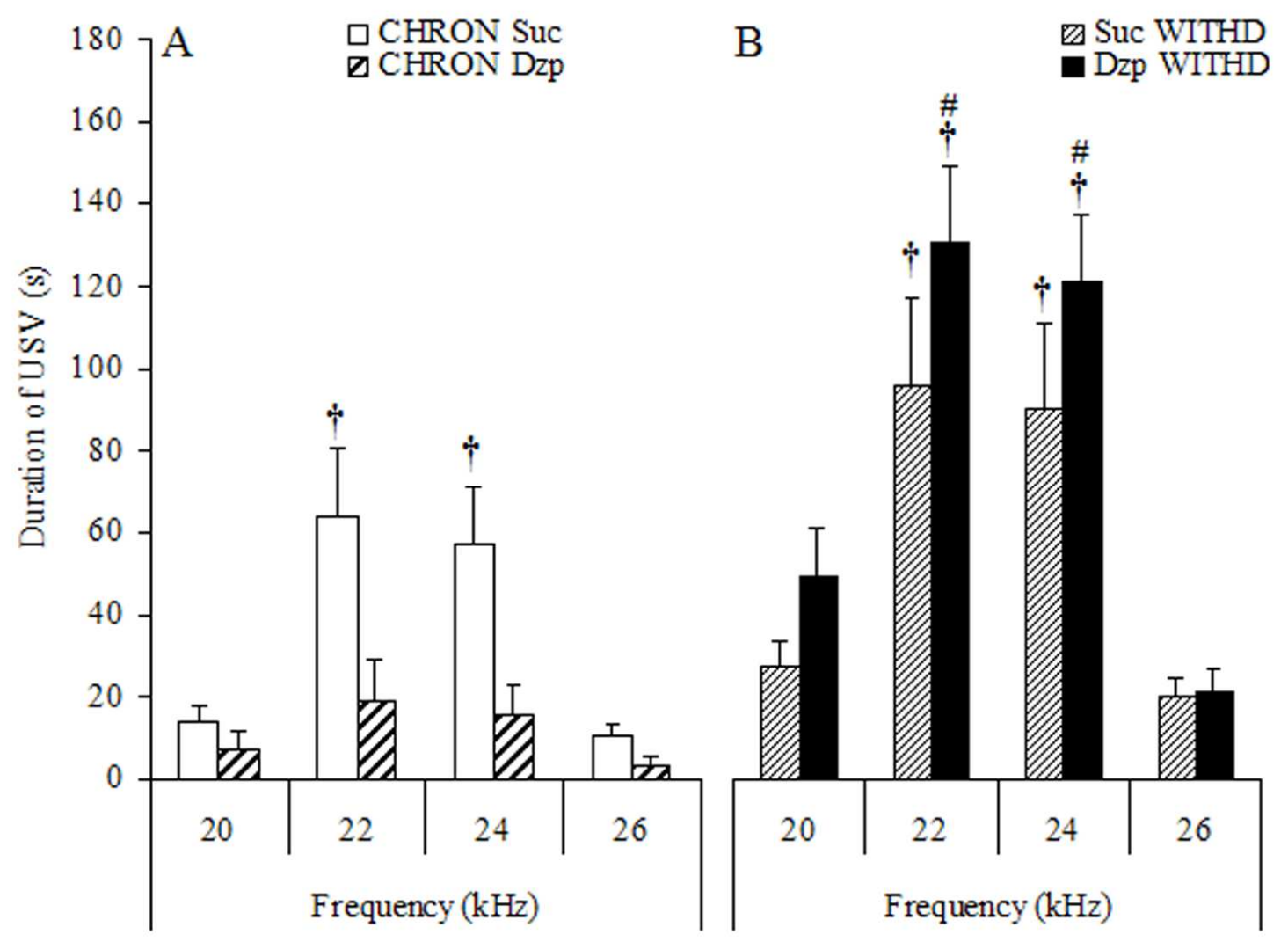

Figure 3: Mean (+SEM) duration of vocalizations emitted at the frequencies of $20-26 \mathrm{KHz}$ during the first 10 minutes after foot-shock delivery of rats tested at $30 \mathrm{~min}(3 \mathrm{~A}, \mathrm{CHRON})$ or $48 \mathrm{~h}$ withdrawal $(3 \mathrm{~B}$, WITHD) from sucrose (Suc) or diazepam (Dzp). † $†$ Significant difference between frequencies on the same group (Suc or Dzp) and condition (CHRON or WITHD) when compared with the $20 \mathrm{KHz}$ frequency. \#Significant difference between WITHD or CHRON conditions on the same frequency on the same group (Dzp or Suc). Two-way ANOVA followed by the Newman-Keuls test.

This analysis showed significant differences between treatments on the number (treatments, $\mathrm{F}_{1,26}=15.83, \mathrm{p}<0.0005$; conditions, $\mathrm{F}_{3,78}=19.15, \mathrm{p}<0.0001$; treatments $\times$ conditions, $\mathrm{F}_{3,78}=4.82, \mathrm{p}<0.005$ ) and duration (treatments, $\mathrm{F}_{1,26}=4.95, \mathrm{p}<0.05$; conditions, $F_{3,78}=10.40, p<0.0001$; treatments $\times$ conditions, $\left.F_{3,78}=3.70, p<0.05\right)$ of vocalizations of rats tested 30 minutes after the last chronic intake of sucrose or diazepam solutions. On the other hand, two-way RM ANOVA showed no significant effects of the treatments or interactions between the treatments and frequencies during 
withdrawal. However significant effects were obtained on the frequencies (number of USV, $\mathrm{F}_{3,78}=44.52, \mathrm{p}<0.0001 ;$ duration of USV, $\left.\mathrm{F}_{3,78}=32.58, \mathrm{p}<0.0001\right)$.

Additional comparisons also showed significant differences on the emission of USV of animals belonging to the sucrose group during withdrawal, when compared with the measures obtained in the same rats during the chronic condition (number, $\mathrm{F}_{3,78}$ $=36.65, \mathrm{p}<0.005$; duration, $\left.\mathrm{F}_{1,26}=18.47, \mathrm{p}<0.0001\right)$. A similar pattern of effects was obtained in animals $48 \mathrm{~h}$ from diazepam withdrawal, when compared with the indices collected on the same rats under chronic diazepam effects. In fact, two-way RM ANOVA revealed significant differences in the number (treatments, $F_{1,26}=45.30, p<$ 0.0001; conditions, $\mathrm{F}_{3,78}=26.98, \mathrm{p}<0.0001$; treatments $\times$ conditions, $\mathrm{F}_{3,78}=13.47, \mathrm{p}<$ 0.0001 ) and duration (treatments, $\mathrm{F}_{1,26}=34.09, \mathrm{p}<0.0001$; conditions, $\mathrm{F}_{3,78}=27.15, \mathrm{p}<$ 0.0001 ; treatments $\times$ conditions, $\left.\mathrm{F}_{3,78}=15.49, \mathrm{p}<0.0001\right)$ of USV.

Post-hoc testing showed that animals under the effects of diazepam decreased their ability to emit $22-\mathrm{KHz}$ USV in the presence of aversive foot-shocks, when compared with the sucrose controls, 30 minutes after the last oral consumption of the drug. The results obtained after $48 \mathrm{~h}$ showed no differences between diazepam and sucrose groups on withdrawal. However, diazepam pretreated rats presented an enhancement of the emission of the aversive 22-KHz USV during withdrawal when compared with the measures registered in the same rats tested under diazepam chronic effects.

\subsection{Effects of diazepam chronic intake and withdrawal on startle response and PPI}

The magnitude of the startle reflex and the prepulse modulation of startle in the two groups of rats (sucrose and diazepam) are illustrated in Figure 4. Two-way RM 
ANOVA revealed that there was no significant difference in groups $\left(\mathrm{F}_{1,38}=1.85, \mathrm{p}>\right.$ 0.05). However, statistically significant effects were obtained for conditions (chronic $\times$ withdrawal; $\left.\mathrm{F}_{1,38}=6.87, \mathrm{p}<0.05\right)$.

The treatments $\times$ condition interaction was also significant $\left(\mathrm{F}_{1,38}=5.53, \mathrm{p}<\right.$ 0.05). Post-hoc tests revealed that diazepam treatment does not change the amplitude of startle if the animals are tested 30 minutes (chronic effects) after the last oral administration (4A) but significantly enhances this response if the rats are tested at $48 \mathrm{~h}$ after diazepam withdrawal (panel 4B). Two-way RM ANOVA showed no differences in the prepulse on startle response between the groups tested $\left(\mathrm{F}_{1,38}=1.85, \mathrm{p}>0.05\right)$ or even significant interaction between treatment $\times$ conditions $\left(\mathrm{F}_{1,38}=0.015, \mathrm{p}>0.05\right.$; panels $A$ and $B)$. However a significant difference was achieved on condition $\left(\mathrm{F}_{1,38}=\right.$ $16.41, \mathrm{p}<0.05)$.

Newman-Keuls post-hoc showed that, independent of the treatment employed, animals of both sucrose and diazepam groups present enhanced PPI during withdrawal, when compared with the measures obtained 30 minutes after the last control or drug solutions intake (4C). 


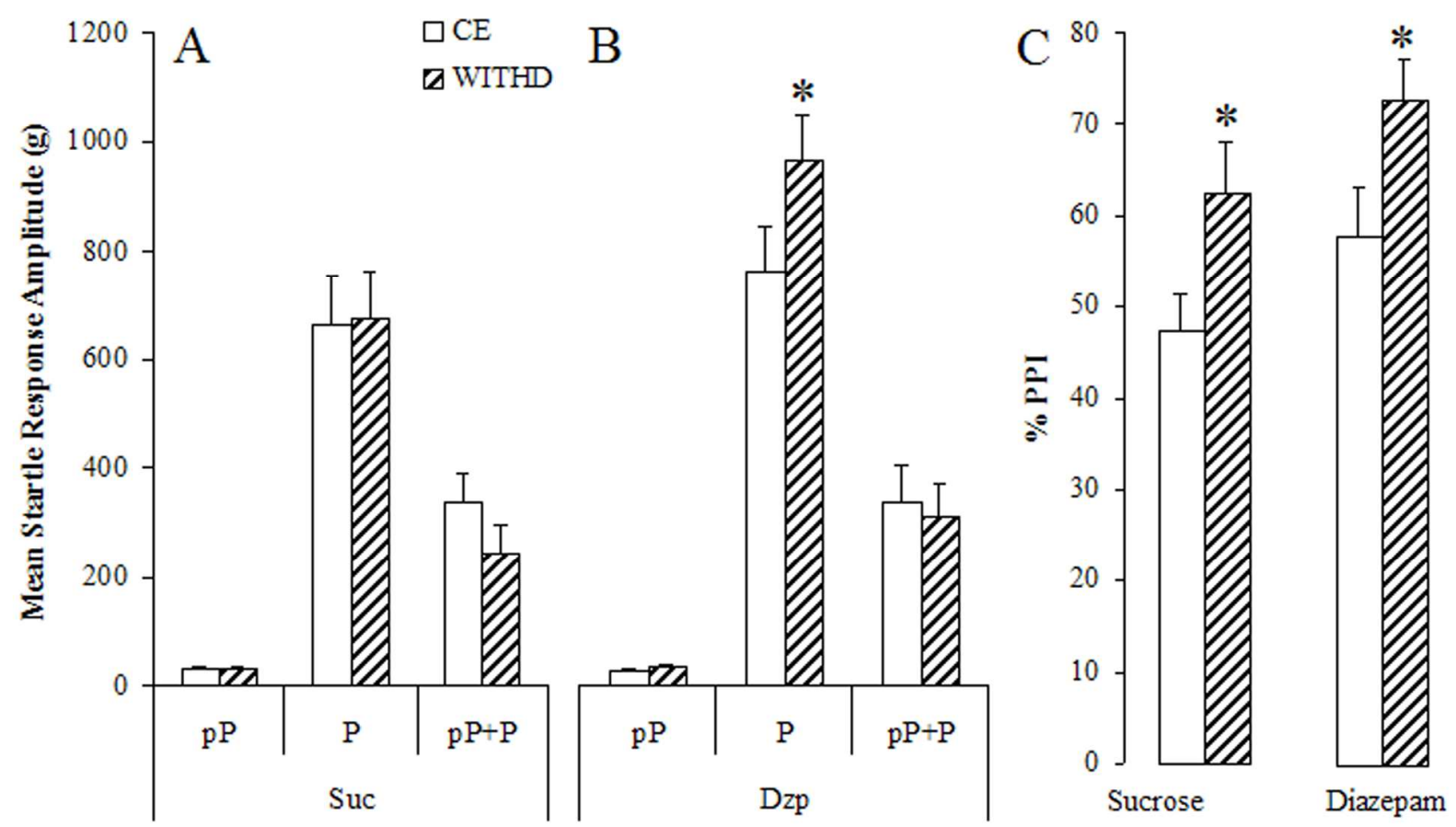

Figure 4: Mean (+SEM) of startle response amplitude of rats chronically treated with sucrose (4A) or diazepam (4B) and tested under chronic effects (CHRON) or during withdrawal (WITHD). Histograms show the amplitude of startle response induced by a prepulse $(\mathrm{pP})$, pulse $(\mathrm{P})$ or prepulse + pulse presentation $(\mathrm{pP}+\mathrm{P})$. The percentage of PPI is showed right $(4 \mathrm{C})$. *Significant difference on startle response amplitude during withdrawal when compared with that observed in rats under chronic sucrose or diazepam effects $(\mathrm{CHRON})$. Data on $\mathrm{pP}, \mathrm{P}$ and $\mathrm{pP}+\mathrm{P}$ were analysed by a two-way RM ANOVA. For the percentage of PPI one-way RM ANOVA was used. The tests were followed by Newman-Keuls post-hoc.

\section{Discussion}

The interruption of a long period of treatment with Bzp produces a great probability of developing withdrawal symptoms both in humans and in laboratory animals (Ladewig, 1984; Lukas and Griffiths, 1982). In fact, high anxiety levels have been reported as a common symptom in patients withdrawn from Bzp and avoidance of these unpleasant symptoms seems to negatively reinforce continuous Bzp use, one of the main components of craving for the drug (Busto et al., 1986; Busto and Sellers, 1991). An increase in anxiety-like states is also observed in rats undergoing 
spontaneous diazepam withdrawal and submitted to several types of animal models of anxiety (Emmett-Oglesby et al., 1983a, 1983b; File, 1990; Greenblatt and Shader, 1974).

As anxiety remains one of the main symptoms present during diazepam withdrawal syndrome, the EPM has frequently been employed to evaluate the degree of drug withdrawal-induced anxiety (Begg et al., 2005; Izzo et al., 2001; Martijena et al., 1996). The EPM was first validated as a test of anxiety-related behaviour for rats (Pellow and File, 1986) and it is frequently considered as a 'primer' test for measuring this type of behaviour. In our study, the interruption of the chronic oral regimen of diazepam caused an anxiogenic effect in the EPM test in the same fashion as those previously reported after abrupt interruption of alcohol chronic treatment (Cabral et al., 2006). In fact, withdrawn animals tend to spend more time inside and also to enter less times into the open arms of the EPM, when compared with controls. Thus, diazepam withdrawal induced anxiety-like effects that remain at least $48 \mathrm{~h}$ after the interruption of the treatment. On the other hand, anxiolytic-like effects associated with an increase in the percentage of time spent in the open arms of the maze was observed in rats under diazepam effects, tested 30 minutes after the last oral intake.

The same pattern of results was obtained in the USV test. There was a reduction of emission of 22-KHz USV elicited by foot-shocks in rats under diazepam effects and an increase in this measure on $48 \mathrm{~h}$ withdrawal. Our results lend further support for the hypothesis that rats exposed to unavoidable stressful stimuli display a series of behavioural and physiological responses, often including intense $22-\mathrm{KHz}$ USV that serves as cries of distress (Cuomo et al., 1988). That this type of response reflects an aversive emotional state relies on the fact that drugs that commonly reduce anxiety also promote anxiolytic-like effects in this model (Cuomo et al., 1988; Vivian and Miczek, 
1993). Moreover, it is well established that such vocalizations serve as an alarm signal warning conspecifics of the presence of imminent danger (Blanchard et al., 1991) as rats submitted to pure $22 \mathrm{KHz}$ tone (Nobre and Brandão, 2004) or playback of $22 \mathrm{KHz}$ (Brudzynski and Chiu, 1995) display characteristic defensive behaviour.

The effects of diazepam withdrawal on ultrasound emissions in rodents were previously demonstrated in some studies that used acoustic stimuli and foot-shocks as ultrasound-elicited stimuli, mainly using the subcutaneous or intraperitoneal injections as the method of drug delivery (Kikusui et al., 2001; Miczek and Vivian, 1993; Nielsen and Sanchez, 1995; Vivian et al., 1994). As it was observed that the chronic intraperitoneal injection of a placebo solution elicits, by itself, anxiety in rats tested in the EPM (Griebel et al., 1994), in this study we used the voluntary oral intake as the method of choice. In a previous study we have demonstrated that this method elicits low anxiety levels in rats treated chronically (Fontanesi et al., 2007). Our results showed higher levels of aversion in withdrawn rats when compared with controls, revealed by their increased levels of vocalizations. Interestingly, there was an increase in the 22$\mathrm{KHz}$ USV in control animals treated with sucrose solutions when they were tested $48 \mathrm{~h}$ after the first session. This effect is probably due to the contextual fear conditioning these animals experienced as they returned to the same experimental box where they previously received foot-shock.

The acoustic startle response is a reflexive neurobehavioural response mediated at the brain stem level (Koch, 1999; Lee et al., 1996) and is known to be affected by the withdrawal from chronic diazepam treatment (Chugh et al., 1992; Miczek and Vivian, 1993; Rasmussen et al., 1993). In our study rats withdrawn from oral diazepam intake increased their magnitude of startle response, in a similar manner to that verified during withdrawal from other drugs of abuse, such as alcohol (Chester et al., 2004; Pohorecky 
et al., 1976), morphine (Harris and Gewirtz, 2004; Kalinichev and Holtzman, 2003) and nicotine (Acri, 1994; Helton et al., 1993).

The results on PPI showed that rats undergoing diazepam treatment or withdrawn from this drug present a similar percentage of inhibition of startle response, when compared with the sucrose controls. The same pattern of effects was observed in other studies that showed no influence of drug withdrawal on the processing of sensory information (Abduljawad et al., 1997; Cabral et al., 2006; Fendt and Mucha, 2001; Peleg-Raibstein et al., 2006). However, there was an unexpected enhancement of the PPI during withdrawal in both sucrose and diazepam groups. As no differences were observed between treatments it is possible that the PPI enhancement during withdrawal in both control and diazepam groups reflects the effects of repetition of the test.

Prepulse inhibition has been hypothesized to be automatic, or 'hard-wired', because it is unlearned (Graham et al., 1975), requires only midbrain and lower brain structures (Leitner and Cohen, 1985), occurs in decorticate animals (Davis and Gendelman, 1977) and also occurs in human adults while they are asleep (Silverstein et al., 1980). However, some studies have initiated some controversy on the automatic nature of the prepulse inhibition. In fact, evidence from studies demonstrated that, in humans, the short-lead interval PPI effect can also be modulated by selective attention, suggesting that it is not an entirely automatic process in all situations (DelPezzo and Hoffman, 1980; Hackley et al., 1987; Hackley and Graham, 1987). Moreover, other studies hypothesized that the accuracy of discriminating the prepulse stimulus is highly correlated to the degree of suppression of the startle reflex (Filion and Cirrani, 1994; Mussat-Whitlow and Blumenthal, 1997; Norris and Blumenthal, 1995).

In a recent study, Zou et al. (2007) showed that the magnitude of the startle reflex response could be strongly inhibited when a prepulse stimulus, that becomes a 
conditioned signal for informing aversive events, is presented immediately before the startling-eliciting stimulus. In addition, the possibility that the prepulse inhibition phenomenon can be modulated by selective attention in some situations relies on the fact that the impairment of some prosencephalic structures, such as the prefrontal cortex or amygdala, mainly involved in the modulation of the cognitive and emotional aspects of behaviour, significantly reduce PPI in rodents (Bubser and Koch, 1994; Koch and Bubser, 1994; Li and Shao, 2003; Swerdlow et al., 1995, 2001). In our study, the increased inhibition of the startle reflex observed on the withdrawal condition in both sucrose- or diazepam-withdrawn animals, seems to be an effect of the aversive learning induced on animals that were submitted repeatedly to the same experimental condition. In this situation, the prepulse could be conditioned to informing the auditory-startling aversive stimulus, promoting an enhancement on the effects of the prepulse, in the same way as a conditioned aversive stimulus, such as foot-shocks can do (Zou et al., 2007). The trends towards difference between diazepam pretreated and sucrose animals observed during withdrawal could be solely due to the high level of anxiety present in withdrawn rats that favour the aversive conditioning in these animals.

In summary, the present results raise some important points in the field of the neurobiology of benzodiazepine withdrawal. It is possible that the same neurobiological substrates that are activated to promote anxiety-like behaviours induced by external aversive stimuli could be overactive during benzodiazepine withdrawal. In a recent study (Fontanesi et al., 2007) we showed high levels of neural activation in brainstem areas mainly involved in the modulation/expression of defensive behaviours in rats withdrawn from diazepam. Chemical or electrical stimulation of these same regions elicits a pattern of performance (Brandão et al., 2003, 2005) similar to that which we obtained in the present study: decreased entries and time spent in the open arms of the 
plus-maze; enhanced emission of ultrasonic vocalizations on $22-\mathrm{KHz}$ aversive frequency; and increased amplitude of startle response. In this case, our results indicate the possibility that the anxiety-like behaviour promoted by withdrawal from diazepam could be the result of alterations in primitive systems that regulates the expression of fear behaviours early, with little or no influence of the prosencephalic structures that modulate cognitive processes.

Finally, the fact that similar emotional negative states are attenuated by anxiolytic drugs seems to support the view that similar neurobiological substrates may be affected by both experiences, anxiety-like states elicited by fear stimuli and drug withdrawal. The fact that prepulse inhibition was not changed in withdrawal animals reinforces the idea that brain structures involved in the processing of sensory and cognitive information have little or no influence in this state.

\section{Conclusions}

Our results confirm previous reports showing that the abrupt interruption of the chronic intake of diazepam elicits high levels of aversion in rats, as revealed in all anxiety tests used in this study. A similar pattern of defensive behaviours is also observed in animals promptly faced with dangerous situations, such as exposure to a predator or cues signalling an aversive event, showing that diazepam withdrawal and fear-elicited stimuli could activate the same neurobiological substrates without harming the processing of sensory and cognitive information. 


\section{Acknowledgements}

Research supported by grants from FAPESP (Proc. $n^{\circ}$ 04/02859-0). 


\section{References}

Abduljawad KA, Langley RW, Bradshaw CM, Szabadi E (1997). Effects of clonidine and diazepam on the acoustic startle response and on its inhibition by prepulses' in man. J Psychopharmacol; 11: 29-34.

Acri JB (1994). Nicotine modulates effects of stress on acoustic startle reflexes in rats: dependence on dose, stressor and initial reactivity. Psychopharmacology (Berl); 116: 255-265.

Anseloni VZ, Brandão ML (1997). Ethopharmacological analysis of behaviour of rats using variations of the elevated plus-maze. Behav Pharmacol; 8: 533-540.

Ashton H (2005). The diagnosis and management of benzodiazepine dependence. Curr Opin Psychiatry; 18: 249-255.

Bassi GS, Nobre MJ, Carvalho MC, Brandão ML (2007). Substance P injected into the dorsal periaqueductal gray causes anxiogenic effects similar to the long-term isolation as assessed by ultrasound vocalizations measurements. Behav Brain Res; 182: 301-307.

Begg DP, Hallam KT, Norman TR (2005). Attenuation of benzodiazepine withdrawal anxiety in the rat by serotonin antagonists. Behav Brain Res; 161: 286-290.

Blanchard RJ, Blanchard DC, Agullana R, Weiss SM (1991). Twenty-two KHz alarm cries to presentation of a predator, by laboratory rats living in visible burrow systems. Physiol Behav; 50: 967-972. 
Brandão ML, Troncoso AC, de Souza Silva A, Huston JP (2003). The relevance of neuronal substrates of defense in the midbrain tectum to anxiety and stress: empirical and conceptual considerations. Eur J Pharmacol; 463: 225-33.

Brandão ML, Borelli KG, Nobre MJ, Santos JM, Albrechet-Souza L, Oliveira AR et al (2005). Gabaergic regulation of the neural organization of fear in the midbrain tectum. Neurosci Biobehav Rev; 29: 1299-311.

Brudzynski SM, Chiu EM (1995). Behavioural responses of laboratory rats to playback of $22 \mathrm{KHz}$ ultrasonic calls. Physiol Behav; 57: 1039-1044.

Bubser M, Koch M (1994). Prepulse inhibition of the acoustic startle response of rats is reduced by 6-hydroxydopamine lesions of the medial prefrontal cortex. Psychopharmacology (Berl); 113: 487-492.

Busto U, Sellers EM, Naranjo CA, Cappell HD, Sanchez-Craig M, Simpkins J (1986). Patterns of benzodiazepine abuse and dependence. Br J Addict; 81: 87-94.

Busto UE, Sellers EM (1991). Anxiolytics and sedative/hypnotics dependence. Br J Addict; 86: 1647-1652.

Cabral A, Isoardi N, Salum C, Macedo CE, Nobre MJ, Brandão ML et al (2006). Fear state induced by ethanol withdrawal may be due to the sensitization of the neural substrates of aversion in the dPAG. Exp Neurol; 200: 200-208.

Chester JA, Blose AM, Froehlich JC (2004). Acoustic startle reactivity during acute alcohol withdrawal in rats that differ in genetic predisposition toward alcohol drinking: effect of stimulus characteristics. Alcohol Clin Exp Res; 28: 677-687. 
Chugh Y, Saha N, Sankaranarayanan A, Sharma PL (1992). Effect of peripheral administration of cinnarizine and verapamil on the abstinence syndrome in diazepam-dependent rats. Psychopharmacology (Berl); 106: 127-130.

Cuomo V, Cagiano R, De Salvia MA, Maselli MA, Renna G, Racagni G (1988). Ultrasonic vocalization in response to unavoidable aversive stimuli in rats: effects of benzodiazepines. Life Sci; 43: 485-491.

Davis M, Gendelman PM (1997). Plasticity of the acoustic startle response in the acutely decerebrate rat. J Comp Physiol Psychol; 91: 549-563.

De Vry J, Benz U, Schreiber R, Traber J (1993). Shock-induced ultrasonic vocalization in young adult rats: a model for testing putative anti-anxiety drugs. Eur J Pharmacol; 249: 331-339.

DelPezzo EM, Hoffman HS (1980). Attentional factors in the inhibition of a reflex by a visual stimulus. Science; 210: 673-674.

Emmett-Oglesby MW, Spencer DG Jr, Lewis M, Elmesallamy F, Lal H (1983a). Anxiogenic aspects of diazepam withdrawal can be detected in animals. Eur $\mathbf{J}$ Pharmacol; 92: 127-130.

Emmett-Oglesby MW, Spencer DG Jr, Elmesallamy F, Lal H (1983b). The pentylenetetrazol model of anxiety detects withdrawal from diazepam in rats. Life Sci; 33: 161-168.

Fendt M, Mucha RF (2001). Anxiogenic-like effects of opiate withdrawal seen in the fear-potentiated startle test, an interdisciplinary probe for drug-related motivational states. Psychopharmacology (Berl); 155: 242-250. 
File SE (1990). The history of benzodiazepine dependence: a review of animal studies. Neurosci Biobehav Rev; 14: 135-146.

File SE, Andrews N (1991). Low but not high doses of buspirone reduce the anxiogenic effects of diazepam withdrawal. Psychopharmacology (Berl); 105: 578-582.

Filion DL, Cirrani M (1994). The function significance of prepulse inhibition: a test of the protection of processing theory. Psychophysiology; 31: S46.

Fontanesi LB, Ferreira R, Cabral A, Castilho VM, Brandão ML, Nobre MJ (2007). Brainstem areas activated by diazepam withdrawal as measured by Fos-protein immunoreactivity in rats. Brain Res; 1166: 35-46.

Geyer MA, Ellenbroek B (2003). Animal behavior models of the mechanisms underlying antipsychotic atypicality. Prog Neuropsychopharmacol Biol Psychiatry; 27: 1071-1079.

Graham FK (1975). The more or less startling effects of weak pre-stimulation. Psyschophysiology; 12: 238-248.

Graham FK, Putnam LE, Leavitt LA (1975). Lead-stimulation effects on human cardiac orienting and blink reflexes. J Exp Psychol; 104: 161-169.

Greenblatt DJ, Shader RI (1974). Drug therapy. Benzodiazepines (second of two parts). N Engl J Med; 291: 1239-1243.

Griebel G, Moreau JL, Jenck F, Misslin R, Martin JR (1994). Acute and chronic treatment with 5-HT reuptake inhibitors differentially modulate emotional responses in anxiety models in rodents. Psychopharmacology (Berl); 113: 463-470. 
Hackley SA, Graham FK (1987). Effects of attending selectively to the spatial position of reflex-eliciting and reflex-modulating stimuli. J Exp Psychol Hum Percept Perform; 13: 411-424.

Hackley SA, Woldorff M, Hillyard SA (1987). Combined use of microreflexes and event-related brain potentials as measures of auditory selective attention. Psychophysiology; 24: 632-647.

Harris AC, Gewirtz JC (2004). Elevated startle during withdrawal from acute morphine: a model of opiate withdrawal and anxiety. Psychopharmacology (Berl); 171: 140147.

Helton DR, Modlin DL, Tizzano JP, Rasmussen K (1993). Nicotine withdrawal: a behavioral assessment using schedule controlled responding, locomotor activity, and sensorimotor reactivity. Psychopharmacology (Berl); 113: 205-210.

Hoffman HS, Ison JR (1980). Reflex modification in the domain of startle: I. Some empirical findings and their implications for how the nervous system processes sensory input. Psychol Rev; 87: 175-189.

Izzo E, Auta J, Impagnatiello F, Pesold C, Guidotti A, Costa E (2001). Glutamic acid decarboxylase and glutamate receptor changes during tolerance and dependence to benzodiazepines. Proc Natl Acad Sci USA; 98: 3483-3488.

Kalinichev M, Holtzman SG (2003). Changes in urination/defecation, auditory startle response, and startle-induced ultrasonic vocalizations in rats undergoing morphine withdrawal: similarities and differences between acute and chronic dependence. J Pharmacol Exp Ther; 304: 603-609. 
Kikusui T, Takeuchi Y, Mori Y (2001). Pharmacological manipulations of the extinction process of fear-induced ultrasonic vocalization in rats. J Vet Med Sci; 63: $591-595$.

Koch M, Bubser M (1994). Deficient sensorimotor gating after 6-hydroxydopamine lesion of the rat medial prefrontal cortex is reversed by haloperidol. Eur J Neurosci; 6: 1837-1845.

Koch M (1999). The neurobiology of startle. Prog Neurobiol; 59: 107-128.

Lacerra C, Martijena ID, Bustos SG, Molina VA (1999). Benzodiazepine withdrawal facilitates the subsequent onset of escape failures and anhedonia: influence of different antidepressant drugs. Brain Res; 819: 40-47.

Ladewig D (1984). Dependence liability of the benzodiazepines. Drug Alcohol Depend; 13: $139-149$.

Lee Y, Lopez DE, Meloni EG, Davis M (1996). A primary acoustic startle pathway: obligatory role of cochlear root neurons and the nucleus reticularis pontis caudalis. J Neurosci; 16: 3775-3789.

Leitner DS, Cohen ME (1985). Role of the inferior colliculus in the inhibition of acoustic startle in the rat. Physiol Behav; 34: 65-70.

Li L, Shao F (2003). Impaired auditory sensorimotor gating: an animal model of schizophrenia. Chin Sci Bull; 48: 2031-2037.

Lukas SE, Griffiths RR (1982). Precipitated withdrawal by a benzodiazepine receptor antagonist (Ro 15-1788) after 7 days of diazepam. Science; 217: 1161-1163. 
Markou A, Kosten TR, Koob GF (1998). Neurobiological similarities in depression and drug dependence: a self-medication hypothesis. Neuropsychopharmacology; 18: 135174.

Marks J (1978). The Benzodiazepines: Use, Oversuse, Misuse, Abuse. 2nd ed. Lancaster: MTP Press.

Martijena ID, Tapia M, Molina VA (1996). Altered behavioral and neurochemical response to stress in benzodiazepine-withdrawn rats. Brain Res; 712: 239-244.

Miczek KA, Vivian JA (1993). Automatic quantification of withdrawal from 5-day diazepam in rats: ultrasonic distress vocalizations and hyperreflexia to acoustic startle stimuli. Psychopharmacology (Berl); 110: 379-382.

Mussat-Whitlow BJ, Blumenthal TD (1997). Impact of acoustic and vibro-tactile prepulse on acoustic and electrical blink reflexes: startle inhibition and task accuracy results. Psychophysiology; 34: S66.

Nielsen CK, Sanchez C (1995). Effect of chronic diazepam treatment on foot-shockinduced ultrasonic vocalization in adult male rats. Pharmacol Toxicol; 77: 177-181.

Nobre MJ, Brandão ML (2004). Analysis of freezing behavior and ultrasonic vocalization in response to foot-shocks, ultrasound signals and GABAergic inhibition in the inferior colliculus: effects of muscimol and midazolam. Eur Neuropsychopharmacol; 14: 45-52.

Nobre M, Sandner G, Brandão ML (2003). Enhancement of acoustic evoked potentials and impairment of startle reflex induced by reduction of GABAergic control of the neural substrates of aversion in the inferior colliculus. Hear Res; 184: 82-90. 
Norris CM, Blumenthal TD (1995). Evidence for the protection of preattentive processing during inhibition of the acoustic startle response. Psychophysiology; 32: S57.

Nunes Mamede Rosa ML, Nobre MJ, Ribeiro OA, Brandão ML (2005). Isolationinduced changes in ultrasonic vocalization, fear-potentiated startle and prepulse inhibition in rats. Neuropsychobiology; 51: 248-255.

Owen RT, Tyrer P (1983). Benzodiazepine dependence. A review of the evidence. Drugs; 25: 385-398.

Peleg-Raibstein D, Sydekum E, Russig H, Feldon J (2006). Withdrawal from continuous amphetamine administration abolishes latent inhibition but leaves prepulse inhibition intact. Psychopharmacology (Berl); 185: 226-239.

Pellow S, File SE (1986). Anxiolytic and anxiogenic drug effects on exploratory activity in an elevated plus-maze: a novel test of anxiety in the rat. Pharmacol Biochem Behav; 24: 525-529.

Petursson H, Lader MH (1981a). Benzodiazepine dependence. Br J Addict; 76: 133145.

Petursson H, Lader MH (1981b). Withdrawal from long-term benzodiazepine treatment. Br Med J (Clin Res Ed); 283: 643-645.

Pohorecky LA, Cagan M, Brick J, Jaffe SL (1976). The startle response in rats: effect of ethanol. Pharmacol Biochem Behav; 4: 311-316. 
Rasmussen K, Helton DR, Berger JE, Scearce E (1993). The CCK-B antagonist LY288513 blocks effects of diazepam withdrawal on auditory startle. Neuroreport; 5: 154-156.

Rosebush PI, Mazurek MF (1996). Catatonia after benzodiazepine withdrawal. J Clin Psychopharmacol; 16: 315-319.

Silverstein LD, Graham FK, Calloway JM (1980). Preconditioning and excitability of the human orbicularis oculi reflex as a function of state. Electroencephalogr Clin Neurophysiol; 48: 406-417.

Swerdlow NR, Geyer MA, Braff DL (2001). Neural circuit regulation of prepulse inhibition of startle in the rat: current knowledge and future challenges. Psychopharmacology (Berl); 156: 194-215.

Swerdlow NR, Lipska BK, Weinberger DR, Braff DL, Jaskiw GE, Geyer MA (1995). Increased sensitivity to the sensorimotor gating-disruptive effects of apomorphine after lesions of medial prefrontal cortex or ventral hippocampus in adult rats. Psychopharmacology (Berl); 122: 27-34.

Vivian JA, Farrell WJ, Sapperstein SB, Miczek KA (1994). Diazepam withdrawal: effects of diazepam and gepirone on acoustic startle-induced $22 \mathrm{KHz}$ ultrasonic vocalizations. Psychopharmacology (Berl); 114: 101-108.

Vivian JA, Miczek KA (1993). Diazepam and gepirone selectively attenuate either 2032 or $32-64 \mathrm{KHz}$ ultrasonic vocalizations during aggressive encounters. Psychopharmacology (Berl); 112: 66-73. 
Woods JH, Katz JL, Winger G (1987). Abuse liability of benzodiazepines. Pharmacol Rev; 39: 251-413.

Zou D, Huang J, Wu X, Li L (2007). Metabotropic glutamate subtype 5 receptors modulate fear-conditioning induced enhancement of prepulse inhibition in rats. Neuropharmacology; 52: 476-486. 
